\title{
Case report of gastric antral vascular ectasia (GAVE)*
}

\author{
Fengli Liu ${ }^{1}$, Feng $\mathrm{Ji}^{1 \#}, \mathrm{Xi} \mathrm{Jin}^{1}$, Yechun Du${ }^{2}$ \\ ${ }^{1}$ Department of Gastroenterology, The First Affiliated Hospital, College of Medicine, Zhejiang University, Hangzhou, China \\ ${ }^{2}$ The Third People's Hospital of Yuhang District, Hangzhou, China \\ Email: \#ifeng1126@sina.com
}

Received 25 March 2013; revised 26 April 2013; accepted 4 May 2013

Copyright (C) 2013 Fengli Liu et al. This is an open access article distributed under the Creative Commons Attribution License, which permits unrestricted use, distribution, and reproduction in any medium, provided the original work is properly cited.

\begin{abstract}
Gastric antral vascular ectasia (GAVE) is a rare clinical disease which can cause recurrent upper gastrointestinal (GI) tract bleeding. It is responsible for about $4 \%$ of non-variceal upper GI haemorrhages. As one of the vascular diseases that happened in stomach, GAVE was also named as watermelon stomach (WS) for its characteristic endoscopic performance. Compared with portal hypertensive gastropathy (PHG) which with similar performances under endoscopy but often appeared as the result of portal hypertension, the etiology of GAVE is unknown. Recent years with further research it received several presumptions according to its histopathological features. Here we will introduce the disease systematically through two cases.
\end{abstract}

Keywords: Gastric Antral Vascular Ectasia (GAVE); Endoscopic Performance; Etiology; Treatments

\section{INTRODUCTION}

Gastric antral vascular ectasia (GAVE) is a rare clinical disease which can cause recurrent upper gastrointestinal (GI) tract bleeding with characteristic endoscopic and histological features. GAVE was first described in 1953 by Rider [1] as "fiery red adherent old blood and scattered areas of profuse bleeding" under endoscopy when he performed gastroscopy to an elderly woman with chronic iron deficiency anemia. The histological feature of this patient was considered erosive type of gastritis with marked veno-capillary ectasia. In 1984, through observing 3 patients, Jabbari [2] described this characteristic appearance of gastric antral under endoscopy as: longitudinal rugal folds traversing the antrum and converg-

\footnotetext{
*Author Contributions: F. Ji designed and performed the operation; Y. C. Du provided patient 2's data; F. L. Liu analyzed the data and wrote the paper; $X$. Jin revised the paper.

${ }^{\#}$ Corresponding author.
}

ing on the pylorus, each containing a visible convoluted column of vessels. This appearance resembled the stripes on a watermelon, so he dubbed the condition watermelon stomach (WS). Clinically, GAVE is often coexistent with other disorders. For instance, 30\% - 66\% GAVE patients also have liver cirrhosis while the incidence of autoimmune connective tissue disorders is more higher for about $62 \%$, among which $31 \%$ for Raynaud's phenomenon and $20 \%$ for sclerodactyly. Other diseases had also been described in GAVE patients, including bone marrow transplantation, chronic renal failure, SLE, ischaemic heart disease, valvular heart disease, hypertension, familial Mediterranean fever, hypothyroidism, diabetes, hypergastrinemia, acute myeloid leukaemia, and so on [3-6]. There are many causes for GI tract bleeding in patients with cirrhosis, and portal hypertensive gastropathy (PHG) and GAVE are both diseases based on mucosal lesions. It is difficult to distinguish them under endoscopy for sometimes they have similar performance, but they are distinct entities, while the pathophysiological mechanisms and treatment of them are completely different. Therefore, it is vitally important to distinguish between them. PHG is mainly the result of increased portal pressure, but the etiology of GAVE is unknown. As GAVE is often associated with other diseases, whether it is an independent disease or comorbidities is not yet clear [7]. Here we introduce the disease systematically through two cases we found in clinical work. However, both patients we presented here do not have other comorbidities, which may support the viewpoint that GAVE was an independent disease. It's a pity that we can't provide details of endoluminal treatment for both of our two patients were sent to surgery after comprehensive assessment.

\section{CASE PRESENTATION}

\subsection{Patient 1}

A 68-year-old woman was initially presented to our out- 
patient clinic with fatigue, dizziness and melena for 2 years. She denied previous history of liver, renal, or autoimmune system disease. Physical examination found nothing special except for appearance of anemia and mild tenderness under xiphoid. Laboratory findings showed a hemoglobin of $49 \mathrm{~g} / \mathrm{l}$; a hematocrit of $20 \mathrm{vol} \%$; occult fecal blood test was positive; other examinations like hepatic function, platelet count, prothrombin time, and international normalized ratio were in reference ranges. Contrast computed tomography scan of the abdomen revealed liver abscess but no other significant GI pathology. The endoscopic appearances showed erythematous stripes on the longitudinal rugal folds radiating out from the pylorus (Figures 1(a) and (b)). The patient have medical therapies such as oral iron supplementation and antacid drugs outside hospital for several months, but her faecal occult blood test was continuing positive,

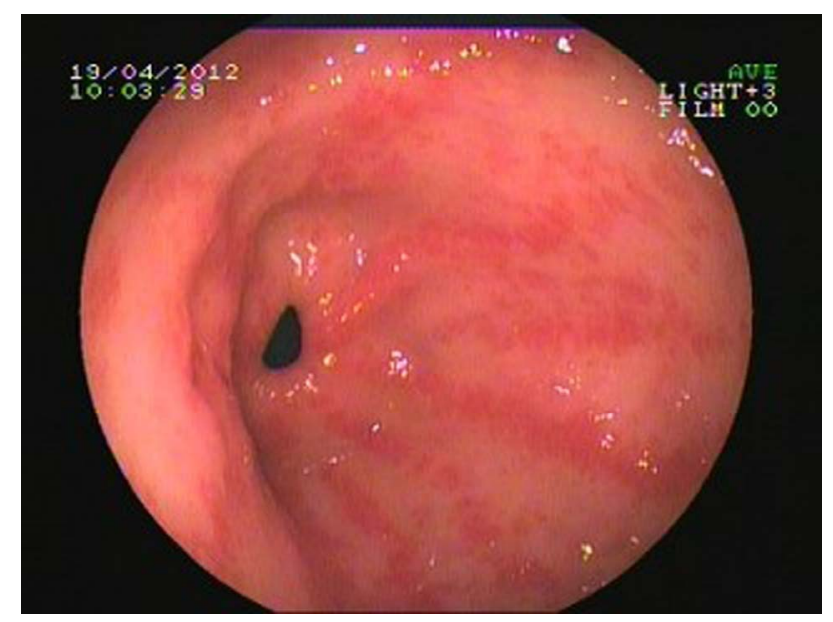

(a)

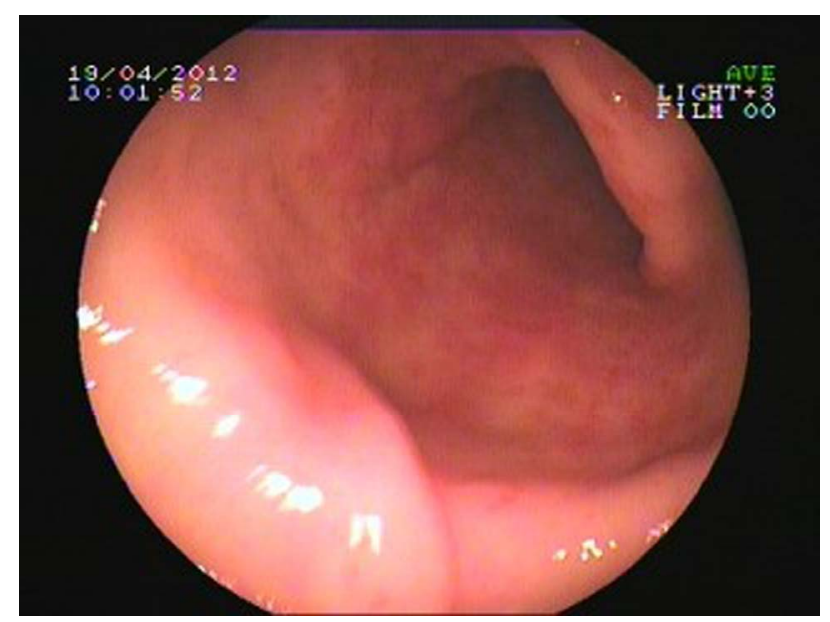

(b)

Figure 1. Endoscopy examination of patient 1. (a) Multiple erythematous streaks traversing the antrum and converging on the pylorus; (b) Each streak contains a visible convoluted column of vessel, but the mucosa between them is smooth and blanched with pressure. monitoring of hemoglobin without elevated, around the first endoscopy found such a serious lesion and involved level of $55 \mathrm{~g} / \mathrm{l}$. Endoscopist considered that the patient's too large area of the stomach, what's more she had no history of other comorbidities which are favorable condition for surgery. Therefore, after blood transfusion the patient underwent an uncomplicated subtotal gastrictomy. The histological features of specimen after surgery showed: (gastric antrum and body) mild chronic atrophic antral gastritis, with light-moderate intestinal metaplasia, edema of submucosa interstitial, and vascular ectasia (comply with gastric antral vascular expansion). We followed up for 5 months, the melena symptom disappeared and she has a rising hemoglobin level stabled. At the end of the follow-up, the patient had a haemoglobin concentration of $110 \mathrm{~g} / \mathrm{l}$ and consistently negative faecal occult blood test for five times.

\subsection{Patient 2}

This 61-year-old female patient presented here with recurrent melena and dizzy for 6 years, more serious for 3 months. At the beginning, she just found symptom of soft and tectonic melena about $1-3$ times/d about $100-200$ g each time, and felt dizziness and abdominal pain sometimes. 3 months ago, she went to hospital near her home for aggravated dizziness even syncope. Laboratory findings showed hemoglobin is $36 \mathrm{~g} / \mathrm{l}$. The doctor advised an endoscopy and found hemorrhagic erosive gastritis near antrum. Colonoscopy and abdominal CT found nothing special. Dizziness improved after treatment with acid inhibitor, drug to protect gastric mucosa and blood transfusion, but still had intermittent melena. So she came to our hospital for further examination to found the cause. Bone marrow biopsy cues iron deficiency anemia while physical examination found an appearance of anemia. Laboratory findings showed the hemoglobin is $42 \mathrm{~g} / \mathrm{l}$ and microcytic hypochromic anemia while faecal occult blood test was positive. Ultrasonic examination of abdomen was normal. A second endoscopy showed multiple mucosal erosions around gastric antrum and scattered blood oozing or active bleeding (Figures 2(a) and (b)). Biopsy pathology was (gastric antrum): moderate chronic superficial gastritis, expansion of tortuous vessels can be seen in lamina propria, comply with gastric antral vascular ectasia (Figure 3). Capsule endoscopy showed: gastric antrum erosion with bleeding and no lesions were found in small intestine. She was admitted to hospital to have the surgery of distal gastrectomy + Billroth II anastomosis. The histological performance of specimen confirmed GAVE. Five days after the surgery a repeat hemogram showed $\mathrm{Hb}$ of $101 \mathrm{~g} / \mathrm{l}$. We also followed up for 5 months, the melena symptom and dizziness disappeared and she has a rising hemoglobin level of $118 \mathrm{~g} / \mathrm{l}$. 


\section{DISCUSSION}

GAVE is a rare disease where most patients are elderly females over $70 \mathrm{~s}$. The lesions prefer sites such as gastric

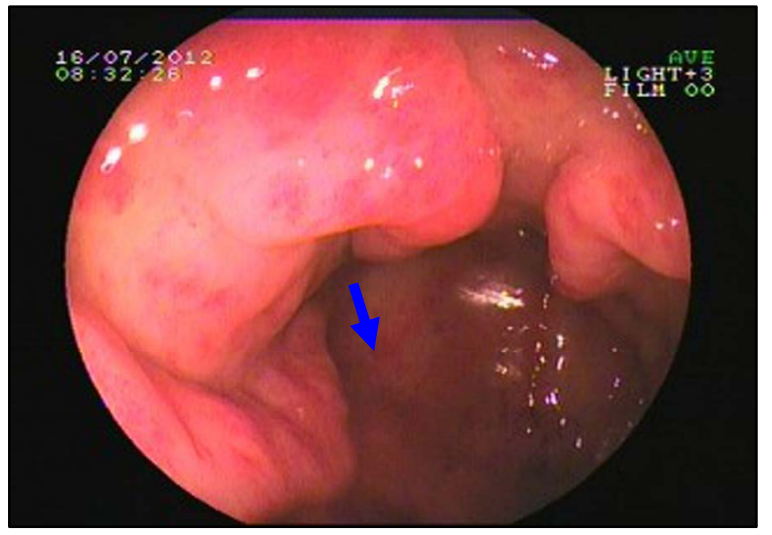

(a)

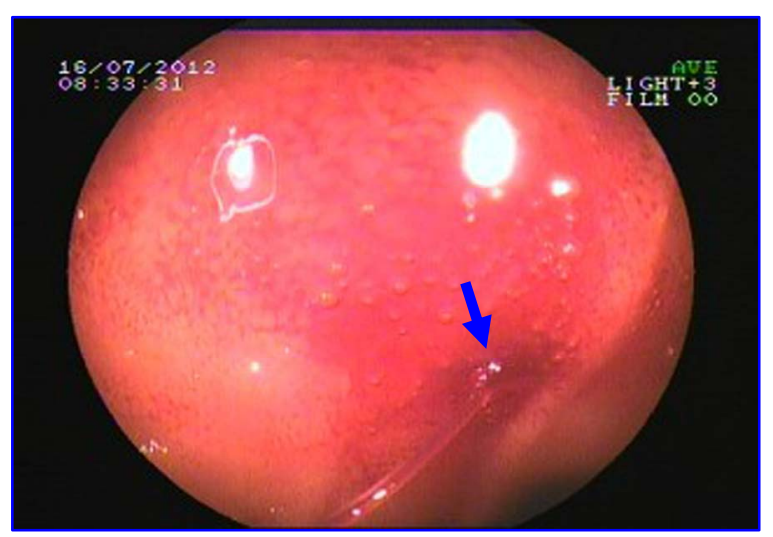

(b)

Figure 2. Endoscopy appearance of patient 2. (a) Visible inflated vessels on the top of longitudinal rugal folds, multiple mucosal erosions can be seen in gastric antrum as we point out with arrow; (b) Mucosal edema and active bleeding (arrow indicating) can be seen in gastric antrum.

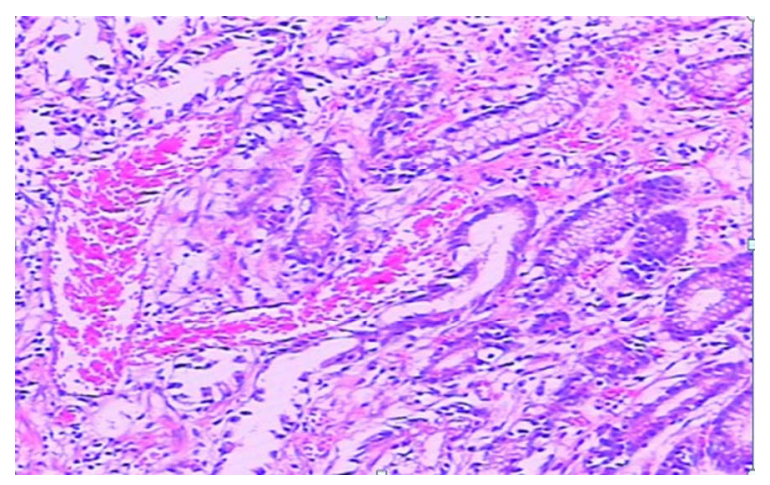

Figure 3. The histological performance of biopsy (gastric antrum): moderate chronic superficial gastritis and several inflated blood vessels can be seen in lamina propria, comply with gastric antral vascular ectasia. pylorus and antrum while involvement of the cardia or proximal stomach have also been reported but rarely $[8,9]$. Statistics show that it is responsible for about $4 \%$ of non-variceal upper GI haemorrhages [10]. Most of the patients began with melena and chronic iron-deficiency anemia clinically. Patients' presentation varies from chronic hidden blood loss to heavy acute GI bleeding, where the latter is rare. At last, patients can develop to significant iron deficiency anemia even transfusion dependent, this increases the risks about blood transfusion, so GAVE was increasingly attracting our attentions as a potential safety concerns. The etiology of WS is unknown. Because of its low incidence, there is a paucity of controlled studies with large sample size of GAVE syndrome about its pathogenesis and most theories are based on single case reports or reports of small series fewer than 15 cases, with sometimes controversial theories. Now the pathophysiological mechanisms about GAVE can be summarized into two categories [10]: some of them insisted that mechanical stress played an important role in the formation of GAVE. They emphasized that gastric antrum abnormal peristaltic waves generated mechanical pressure and caused partial prolapse mucosa of distal gastric when passing through the pyloric ring, with longterm inflammation stimulus the muscular layer proceed fibromuscular hyperplasia, mucosa cumulated into longitudinal folds, vessels gradually bulged and eventually induced the characteristic endoscopic and histological performance of GAVE. Several researchers of them also put forward that most of the GAVE patients' histologic changes include superficial hyperplastic antral mucosa, vascular ectasia, thrombi, spindle cell (smooth muscle cells and fibroblasts) proliferation of the lamina propria, similar to a certain extent with findings of volvulus or mucosal trauma, suggesting that disordered mucosal stress may play a pivotal role in GAVE lesions. Scientists insist another theory that locally high concentrations of vasoactive substances secreted by extra-epithelial and intraepithelial proliferations of neuroendocrine cells lead to the development of expansion of the blood vessels.

Recent years, more and more GAVE cases are reported with the popularity of endoscopy. As an important diagnostic method, the experience of our patient 2 told us that repeated endoscopy is necessary when facing unexplained chronic blood loss patient. Here we summarized typical endoscopic performance of GAVE into two distinctive patterns: The majority of patients including our two cases have antral disease with classic ectatic vascular tissue radiating out from the pylorus that described by Jabbari et al as WS; another type has scattered red patchy or punctuate lesions distributed diffusely throughout the proximal and distal stomach and its predilection sites also located in the antrum [11]. GAVE patients with cirrhotic syndrome tend to have diffuse le- 
sions under endoscopy and most of them are males (75\%) with mean age of 65 years old. Patients without cirrhosis but with GAVE are more likely to have classic WS performance described before, where majority of them are females (71\%) with a relatively higher mean age of 73 years. This is similar to the epidemiologic features in human susceptible to cirrhosis and connective-tissue diseases to a certain extent $[12,13]$. The type with scattered lesions is difficult to distinguish from portal hypertensive gastropathy (PHG) under endoscopy. Although they may lead to similar clinical manifestations, they are two clearly different clinical entities that can cause mucosal lesions. The pathophysiological mechanisms, histopathology, and therapeutic approaches of them are completely different. PHG often appeared as the result of portal hypertension, while the severity of portal pressure is not directly related to GAVE. Whereas GAVE is most commonly limited to the antrum, PHG predominantly involved the mucosa in the fundus and corpus, and with characteristic endoscopic appearance of snake-skin mosaic pattern with or without mucosal erythema. Measures used to control PHG are based on reducing portal pressure, but such treatments have no significant impact on GAVE. Compared with PHG, GAVE patients tend to have higher degree of liver dysfunction, greater blood loss, lower serum gastrin levels and a higher incidence of previous sclerotherapy. The best way to distinguish them is biopsy, particularly in those cases the endoscopic appearance of the lesions can lead to some confusion [14, 15].

According to a long-term history of chronic blood loss, special performance under gastroscopy and histopathological findings, the diagnosis of GAVE is not difficult. The next important question was how to cure it. In many cases of GAVE, treatment of underlying medical conditions or stop the caused performance can lead to resolution of GAVE syndrome even complete disappearance of lesions, such as cirrhosis (by liver transplantation) or scleroderma (by immunomodulatory medications) [3,12, 16]. Ehab Saad Aldin once reported a patient with gastrointestinal stromal tumors (GISTs) was found to have GAVE after 8 months treatment with Imatinib. After discontinuing Imatinib, the patient underwent repeated endoscopy, which showed significant improvement in the stomach's erythema and GAVE [17]. Therapies for GAVE aim to control the symptoms of acute or chronic blood loss, eliminate or reduce transfusion dependence, improve the life qualities of patients. They can be separated into three categories: pharmacologic, endoluminal, and surgical approaches. Pharmacologic therapies so far we used with variable success rates, include tranexamic acid, thalidomide, $\alpha$-interferon, calcitonin, cyproheptadine, corticosteroids, estrogen-progesterone combinations and serotonin antagonist and so on [18-21]. Clinically we use phar- macologic therapy as an auxiliary method for a relatively higher rate of recurrence and lower effectiveness. Since Frager [22] effectively treated GAVE with neodymium-doped yttrium aluminum garnet laser therapy (Nd: YAG), various endoscopic treatments get a repaid development and some of them have been reported to be successfully used, such as sclerotherapy, heater probe, monopolar coagulation, multipolar electrocoagulation, cryotherapy, endoscopic band ligation, endoscopic mucosal resection, and argon plasma coagulation (APC). Generally, APC was considered as the preferred and the most frequently used endoscopic therapy for GAVE up to now, for it was reported to be safer and more effective in comparison to other non-contact treatments with the efficiency ranging from $70 \%-100 \%$. However, the recurrence rate of GAVE was $68.2 \%$ while endoscopic ligation was used as remedies when APC failed [23-25]. Although associated with a high rate of morbidity and mortality (the postoperative mortality rate was $6.6 \%$ to $7.4 \%$ ) when attempted in patients with GAVE especially combined liver cirrhosis and other serious concomitant diseases, surgical resection is still the only reliable and often the last resort method for refractory cases or recurrent GI bleeding. It also applies to patients whose lesions were dispersed and involved a large area of the stomach wall without serious comorbidities (as our cases) [26]. As mentioned above, patients easily got recurrence after drug control and endoscopic treatment, and 2/3 of recurrent people did not have clinical symptoms [12]. Therefore, it is necessary to have a preventive endoscopic treatment and regularly gastroscopic follow-up. Meanwhile, the hemoglobin level can be used as an index to evaluate whether the patient have a latent chronic blood loss and quantity.

In summary, as a rare disorder that can cause upper GI blood loss for a long time, most of GAVE patients presented with repeated melena and chronic anemia, that might be overlooked for there are more other common causes. In clinical, there is a paucity of controlled studies with large sample size on its pathogenesis and comprehensive assessment of the efficacy about various treatments. When facing GAVE patients, we are trapped at the selection of treatments, especially patients with cirrhosis and portal hypertension. Generally speaking, endoscopic therapies such as APC were considered as the preferred treatment. But it's a pity that we can't provide details of endoluminal treatment for both of our two patients had no history of other comorbidities which are favorable condition for surgery, so they were sent to surgery in succession after comprehensive assessment.

\section{REFERENCES}

[1] Rider, J.A., Klotz, A.P. and Kirsner, J.B. (1953) Gastritis 
with veno-capillary ectasia as a source of massive gastric hemorrhage. Gastroenterology, 24, 118-123.

[2] Jabbari, M., Cherry, R., Lough, J.O., et al. (1984) Gastric antral vascular ectasia: The water melon stomach. Gastroenterology, 87, 1165-1170.

[3] Ward, E.M., Raimondo, M., Rosser, B.G., et al. (2004) Prevalence and natural history of gastric antral vascular ectasia (GAVE) in patients undergoing orthoptic liver transplantation. Journal of Clinical Gastroenterology, 38, 898-900. doi:10.1097/00004836-200411000-00013

[4] Tobin, R.W., Hackman, R.C., Kimmey, M.B., et al. (1996) Bleeding from gastric antral vascular ectasia in marrow transplant patients. Gastrointestinal Endoscopy, 44, 223229. doi:10.1016/S0016-5107(96)70155-4

[5] Sebastian, S., O’Morain, C.A. and Buckley, M.J. (2003) Review article: Current therapeutic options for gastric antral vascular ectasia. Alimentary Pharmacology \& Therapeutics, 18, 157-165. doi:10.1046/j.1365-2036.2003.01617.x

[6] Takahashi, T., Takuya, M., Oki, M., et al. (2006) Severe hemorrhage from gastric antral vascular ectasia developed in a patient with AML. International Journal of Hematology, 83, 467-468. doi:10.1532/IJH97.06052

[7] Ripoll, C. and Garcia-Tsao, G. (2010) Management of gastropathy and gastric vascular ectasia in portal hypertension. Clinical Liver Disease, 14, 281-295. doi:10.1016/j.cld.2010.03.013

[8] Stotzer, P.O., Willeri, R. and Kilander, A.F. (2002) Watermelon stomach: Not only an antral disease. Gastrointestinal Endoscopy, 55, 897-900. doi:10.1067/mge.2002.124558

[9] Molina Infante, J., Hernández Alonso, M., Mateos, J.M., et al. (2009) Refractory gastric antral vascular ectasia extending to the proximal stomach. Revista Espanola de Enfermedades Digestivas, 101, 71-80. doi:10.4321/S1130-01082009000100013

[10] Selinger, C.P. and Ang, Y.S. (2008) Gastric antral vascular ectasia (GAVE): An update on clinical presentation, pathophysiology and treatment. Digestion, 77, 131-137. doi:10.1159/000124339

[11] Chaves, D.M., Sakai, P., Oliveira, C.V., et al. (2006) Watermelon stomach: Clinical aspects and treatment with argon plasma coagulation. Arquivos de Gastroenterologia, 43, 191-195. doi:10.1590/S0004-28032006000300007

[12] Fuccio, L., Zagari, R.M., Serrani, M., Eusebi, L.H., Grilli, D., Cennamo, V., Laterza, L., Asioli, S., Ceroni, L. and Bazzoli, F. (2009) Endoscopic argon plasma coagulation for the treatment of gastric antral vascular ectasia-related bleeding in patients with liver cirrhosis. Digestion, 79, 143-150. doi:10.1159/000210087

[13] Ito, M., Uchida, Y., Kamano, S., Kawabata, H. and Nishioka, M. (2001) Clinical comparisons between two subsets of gastric antral vascular ectasia. Gastrointestinal Endoscopy, 53, 764-770. doi:10.1067/mge.2001.113922

[14] Spahr, L., Villeneuve, J.P., Dufresne, M.P., et al. (1999) Gastric antral vascular ectasia in cirrhotic patients: Absence of relation with portal hypertension. Gut, 44, 739742. doi:10.1136/gut.44.5.739
[15] Quintero, E., Pique, J.M., Bombi, J.A., et al. (1987) Gastric mucosal vascular ectasias causing bleeding in cirrhosis. A distinct entity associated with hypergastrinemia and low serum levels of pepsinogen I. Gastroenterology, 93, 10541061.

[16] Vincent, C., Pomier-Layrargues, G., Dagenais, M., Lapointe, R., Letourneau, R., Roy, A., Pare, P. and Huet, P.M. (2002) Cure of gastric antral vascular ectasia by liver transplantation despite persistent portal hypertension: A clue for pathogenesis. Liver Transplantaion, 8, 717720. doi:10.1053/jlts.2002.34382

[17] Aldin, E.S., Mourad, F. and Tfayli, A. (2012) Gastric antral vascular ectasia in a patient with GIST after treatment with imatinib: Case report and literature review. Japanese Journal of Clinical Oncology, 42, 447-450. doi:10.1093/jico/hys032

[18] Dunne, K.A., Hill, J. and Dixon, J.F. (2006) Treatment of chronic transfusion-dependent gastric antral vascular ectasia (watermelon stomach) with thalidomide. European Journal of Gastroenterology \& Hepatology, 18, 455-456. doi:10.1097/00042737-200604000-00024

[19] Disdier, P., Schleinitz, N., Perreard, M., et al. (1995) Dramatic improvement of watermelon stomach with alphainterferon. American Journal of Gastroenterology, 90, 1009-1010.

[20] Tran, A., Villeneuve, J.P., Bilodeau, M., et al. (1999) Treatment of chronic bleeding from gastric antral vascular ectasia (GAVE) with estrogen-progesterone in cirrhotic patients: An open pilot study. American Journal of Gastroenterology, 94, 2909-2911. doi:10.1111/j.1572-0241.1999.01436.x

[21] Cabral, J.E., Pontes, J.M., Toste, M., et al. (1991) Watermelon stomach: Treatment with a serotonin antagonist. American Journal of Gastroenterology, 86, 927-928.

[22] Frager, J.D., Brandt, L.J., Frank, M.S., et al. (1988) Treatment of a patient with watermelon stomach using transendoscopic laser photocoagulation. Gastrointestinal Endoscopy, 34, 134-137. doi:10.1016/S0016-5107(88)71280-8

[23] Jaime Poniachik, T., Zoltán Berger, F. and Araxi Manuguián G. (2012) Endoscopic band ligation, for gastric antral vascular ectasia. Report of two cases. Revista médica de Chile, 140, 364-367.

[24] Kwan, V., Bourke, M.J., Williams, S.J., et al. (2006) Argon plasma coagulation in the management of symptomatic gastrointestinal vascular lesions: Experience in 100 consecutive patients with long-term follow-up. American Journal of Gastroenterology, 101, 58-63. doi:10.1111/j.1572-0241.2006.00370.x

[25] Sato, T., Yamazaki, K. and Akaike, J. (2012) Endoscopic band ligation versus argon plasma coagulation for gastric antral vascular ectasia associated with liver diseases. Journal of Digestive Endoscopy, 24, 237-242. doi:10.1111/j.1443-1661.2011.01221.X

[26] Dulai, G.S. and Jensen, D.M. (2006) Treatment of watermelon stomach. Current Treatment Options in Gastroenterology, 9, 175-180. doi:10.1007/s11938-006-0036-1 